\title{
Review Article \\ Recent Advances in the Pathogenesis of Autoimmune Hair Loss Disease Alopecia Areata
}

\author{
Taisuke Ito \\ Department of Dermatology, Hamamatsu University School of Medicine, 1-20-1 Handayama, Higashi-ku, \\ Hamamatsu 431-1192, Japan \\ Correspondence should be addressed to Taisuke Ito; itoutai@hama-med.ac.jp
}

Received 2 May 2013; Accepted 19 August 2013

Academic Editor: Guixiu Shi

Copyright (C) 2013 Taisuke Ito. This is an open access article distributed under the Creative Commons Attribution License, which permits unrestricted use, distribution, and reproduction in any medium, provided the original work is properly cited.

Alopecia areata is considered to be a cell-mediated autoimmune disease, in which autoreactive cytotoxic $\mathrm{T}$ cells recognize melanocyte-associated proteins such as tyrosinase. This review discusses recent advances in the understanding of the pathogenesis of alopecia areata, focusing on immunobiology and hormonal aspects of hair follicles (HFs). The HF is a unique "miniorgan" with its own immune and hormonal microenvironment. The immunosuppressive milieu of the anagen hair bulb modulated by immunosuppressive factors is known as "hair follicle immune privilege." The collapse of the hair follicle immune privilege leads to autoimmune reactions against hair follicle autoantigens. Alopecia areata is sometimes triggered by viral infections such as influenza that causes excess production of interferons (IFN). IFN- $\gamma$ is one of the key factors that lead to the collapse of immune privilege. This paper reviews the interactions between the endocrine and immune systems and hair follicles in the pathogenesis of alopecia areata.

\section{Introduction}

1.1. Clinical Features. Alopecia areata (AA) is a relatively common disease encountered by dermatologists. Patients with AA represent approximately $0.7 \%$ to $3.8 \%$ of all the patients attending dermatology clinics $[1,2]$. Males and females are affected equally [3]. Disease onset before the 4th decade was reported to be $85.5 \%$ in the Asian population [2].

Based on the extent of hair loss, the hair loss pattern of AA can be described as single delimited patches; patchy $\mathrm{AA}$, in which there is a partial loss of scalp hair; alopecia totalis (AT), in which $100 \%$ of scalp hair is lost; or alopecia universalis (AU), in which there is a $100 \%$ loss of all scalp and body hair [4]. Less common types of AA include reticular patches of hair loss; ophiasis type, a band-like hair loss in the parieto-temporo-occipital area; ophiasis inversus (sisaipho), a rare band-like hair loss in the frontal parietotemporal scalp; and a diffuse thinning of a part or all of the scalp [4]. Another variant named "acute diffuse and total alopecia" is characterized by acute scalp hair loss, extensive involvement with infiltration of eosinophils around hair follicles (HFs), and a favorable prognosis. It was first described by SatoKawamura et al. and was thought to be limited to females [5], but Lew et al. described that is affected male patients in their case series [6].

1.2. Association with Other Autoimmune Diseases. AA may be associated with other autoimmune diseases, particularly thyroid autoimmune disease such as Hashimoto's thyroiditis and Basedow's disease. The prevalence of thyroid disease in patients with AA ranges from $8 \%$ to $28 \%$ [7]. The presence of thyroid autoantibodies does not correlate with AA severity [8], and treatment is not warranted. Vitiligo, an autoimmune skin disease affecting melanocytes, is also associated with AA. The prevalence of vitiligo in AA patients is $3 \%$ to $8 \%$ compared with $1 \%$ in the United States population [9]. These disease associations suggest a relationship between autoimmunity and AA.

1.3. Psychiatric Morbidity. AA is associated with psychiatric morbidity, especially anxiety and depression [10]. In a study of 31 AA patients, Colón et al. reported that $74 \%$ were 
given one or more lifetime psychiatric diagnoses based on structured psychiatric interviews [11]. The lifetime prevalence rates of major depression (39\%) and generalized anxiety disorder (39\%) were particularly high [11]. These studies indicate that patients with AA are at an increased risk for developing anxiety and depression, and psychiatric referral may be warranted in AA patients.

1.4. Histopathology. The accumulation of mononuclear cells in and around hair bulbs-so-called "swarm of bees"-is the most characteristic histopathological change in AA [12]. This is observable particularly in the acute stage of the disease, and it is composed of both CD4+ and CD8+ cells with a high $\mathrm{CD} 4+/ \mathrm{CD} 8+$ ratio in clinically active disease [13]. In the chronic stage, there is a marked HF miniaturization and cell accumulation decreases, but accumulation of CD8+ T cells is still observable [14].

\section{Pathogenesis}

2.1. Genetics. Genetics play an important role in the pathogenesis of AA. For example, monozygotic twins who suffered from AA after mumps infection had a similar disease onset and hair loss patterns [15]. Specific alleles such as DQB1*03 and DRB1* 1104 have been reported as markers of susceptibility to AA [16-20]. The HLA alleles DRB1*1104 (HLA-DR11) and DQB1 ${ }^{*} 0301$ (HLA-DQ7) may be associated with AT/AU [17]. These findings suggest that the onset and progression of AA is associated with specific HLA class II alleles [1821]. Recently, Petukhova et al. [22] performed a genome-wide association study (GWAS) to determine the genetic architecture of AA in a sample of 1,054 AA cases and 3,278 controls using a combination of Illumina $610 \mathrm{~K}$ and $550 \mathrm{~K}$ arrays. The GWAS revealed 139 single nucleotide polymorphisms (SNPs) that are significantly associated with AA $(P \leq 5 \times$ $10^{-7}$ ). Several susceptibility loci for AA were identified, most of which were clustered in eight genomic regions and fell within discrete linkage disequilibrium blocks. These include loci on chromosome 2q33.2 containing CTLA4, chromosome $4 \mathrm{q} 27$ containing $I L-2 / I L-21$, chromosome 6p21.32 containing the HLA, chromosome 6q25.1 harboring the ULBP genes, chromosome 10p15.1 containing IL-2RA (CD25), and chromosome 12q13 containing Eos (IKZF4) and ERBB3. One SNP resides on chromosome 9q31.1 within syntaxin 17 (STX17), and one resides on chromosome 11q13, upstream from peroxiredoxin 5 (PRDX5). The ULBP genes encoding the ligands of the natural killer cell receptor NKG2D reside in a 180-kilobase MHC class I-related cluster on human chromosome 6q25.1. NKG2D is expressed on natural killer (NK) and CD8+ T cells, and is activated by ULBP. The role of the NK cell activating receptor, NKG2D, in the pathogenesis AA will be described later in this review.

2.2. Collapse of Hair Follicle Immune Privilege. One of the most intriguing features of hair biology is the immune privilege of the anagen HF $[23,24]$ that is characterized by an immunosuppressive milieu around the hair bulb. Apart from the anagen HF, other sites of immune privilege include the anterior eye chamber, parts of testis and ovaries, adrenal cortex, parts of the central nervous system enclosed by the blood-brain barrier, the placenta, and the hamster cheek pouch $[23,24]$. The unique microenvironment of these immune-privileged sites protects the organs from deleterious immune reactions and loss of function. For example, severe inflammation of the anterior chamber of the eye could lead to blindness, and an immune reaction in the central nervous system could cause serious brain damage. Although scalp and trunk hair are not necessary for human survival, significant hair loss could be deadly for mammals such as polar bears, seals, and reindeer [25].

The HF immune privilege (IP) is maintained by several factors, including the lack of major histocompatibility complex (MHC) class I in the proximal outer root sheath (ORS) and matrix cells (Table 1). HFIP is present during anagen but is lost during the resting (telogen) and regression (catagen) phases of the hair cycle. Collapse of HFIP is thought to contribute to the development of AA (Figure 1), in which pigment-producing anagen hair bulbs are attacked by inflammatory cells [23]. MHC class I is strongly expressed in AA lesions, raising the possibility that $\mathrm{CD} 8+\mathrm{T}$ cells react to autoantigens by binding to MHC class I molecules. Interferon (IFN)- $\gamma$, a key cytokine implicated in the pathogenesis of AA, upregulates MHC class I expression in cultured HFs in vitro [26]. Virus infections can increase the production of IFN- $\gamma$ in vivo, and it has been reported that the swine flu virus can trigger or exacerbate AA [27].

2.3. Autoantigens. Several studies of AA suggest that melanogenesis-associated peptides expressed by melanin-producing anagen HFs are the key autoantigens targeted by autoreactive cytotoxic $T$ cells $[28,29]$. Possible involvement of melanogenesis-associated autoantigens in AA was suggested by the following observations: sparing of white/greying HFs in AA; regrowing hair shafts are usually white followed by repigmentation, association with vitiligo, and the sudden onset of fulminant AA affecting mostly pigmented HFs (overnight greying). Follicular melanocytes are possible targets in AA. Indeed, follicular melanocytes in AA show both histological and ultrastructural abnormalities [30]. Using the human scalp explant/SCID mouse transfer model, Gilhar et al. demonstrated that melanocyte-associated $\mathrm{T}$ cell epitopes are capable of functioning as autoantigens and result in AA in human scalp grafts [29]. Melanocyte HLA-A2-restricted peptides can activate $\mathrm{T}$ cells for the transfer of $\mathrm{AA}$ to autologous scalp skin grafts on SCID mice, indicating that melanocyte-associated autoantigens can be pathogenic.

2.4. "Swarm of Bees" Linked to Th1 Cytokines, Chemokines, and Chemotaxis. A unique histopathological feature in the acute stage of AA is the dense accumulation of lymphocytes around the hair bulbs-so-called "swarm of bees" - that is a result of the collapse of HFIP with exposure of autoantigens, leading to an accumulation of autoreactive T cells [12]. The mononuclear cells that accumulate in and around the lesional hair bulb consist of $60-80 \%$ CD $4+$ T cells, $20-40 \%$ CD $8+$ T cells, and NK cells $[13,31]$. IFN- $\gamma$, a representative Th1 cytokines, 


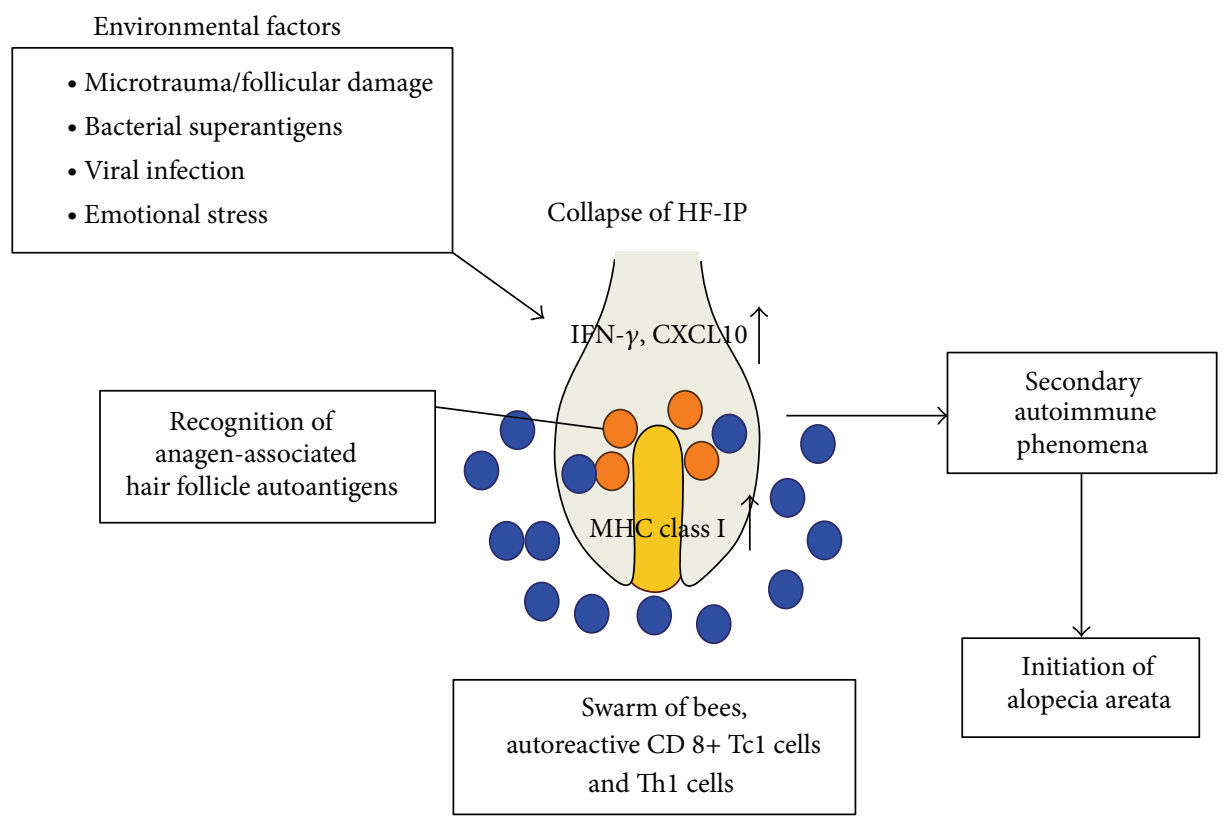

Lymphocytes

HF autoantigens

FIGURE 1: The pathogenesis of alopecia areata and treatment strategies. Environmental factors such as viral infections and bacterial superantigens may induce IFN- $\gamma$ and CXCL10 expressions in the hair bulbs. Subsequently, autoreactive Th1 and Tcl cells (blue circles) accumulate in and around hair bulbs-the so-called "swarm of bees." Anagen-associated hair follicle (HF) autoantigens (orange circles) are recognized by Thl and Tcl cells, which lead to a secondary autoimmune phenomenon and resultant hair loss.

TABLE 1: The mechanism of HF immune privilege.

(i) Absent or barely detectable expression of MHC class I.

(ii) Hair follicular melanocytes of the human anagen scalp are MHC class I-negative.

(iii) Downregulation of the MHC class I pathway-related molecules $\beta 2$-microglobulin and transportation of antigen processing-2 (TAP-2).

(iv) Downregulation of interferon regulatory factor-1 expression.

(v) Upregulation of immunosuppressive factors such as TGF- $\beta 1$ and TGF- $\beta 2$, ACTH, and $\alpha$-MSH.

(vi) Absence of MHC class II+ or NLDC-145+ Langerhans cells.

(vii) Sparse distribution of NK cells and CD4+ and CD8+ T cells.

(viii) Absence of lymphatics.

is prominently expressed in AA lesions and may induce the collapse of HFIP by upregulating MHC class I expression [32]. Autoimmune hair loss similar to AA can be reproduced in $\mathrm{C} 3 \mathrm{H} / \mathrm{HeJ}$ mice by injecting IFN- $\gamma$, which induces the follicular expression of MHC classes I and II [33].

Several studies have examined the expression of chemokines and their receptors in AA. For example, the expression of Th1 chemokines CXCL9/MIG and CXCL10/IP10 is increased in AA lesions [34, 35]. Serum CXCL9 is elevated in AA patients, and its level is correlated with disease activity [36]. Transcriptional profiling revealed that
CXCL10 is highly upregulated in AA lesions compared with nonlesional skin [37]. Our study showed that the proportions of CXCR3+ CD4+ Th1 cells and CXCR3+ CD8+ Tc1 cells are significantly increased in the PBMCs of AA patients [11]. CD8+ T cells can be differentiated into two effector phenotypes, Tc1 and Tc2, which secrete different cytokines [38-40]. CD8+ T cells that secrete IFN- $\gamma$ but not IL-4 and IL-5 are known as type I CD8+ cytotoxic T (Tc1) cells, while those that secrete IL- 4 and IL- 5 but not IFN- $\gamma$ are type II CD8+ cytotoxic T (Tc2) cells. Tc1 cells kill tumor targets by either perforin or Fas-mediated mechanisms, whereas Tc2 cells mainly use the perforin pathway [41]. Tc1 cells are implicated in the development of autoimmune diseases such as experimental autoimmune thyroiditis [42]. In the acute phase AA, the proportion of MAGE-A3 is specific, IFN$\gamma$-producing T cells in PBMCs is increased [11]. Therefore, the increased numbers of CXCR3+ CD8+ Tc1 cells may contribute to the cell-mediated autoimmune reactions in AA.

In addition, we demonstrated that freshly isolated CD4+ and CD8+ T cells from AA patients displayed strong chemotactic activity towards CXCL10 using a real-time chemotaxis assay [14]. The $\mathrm{T}$ cell chemotactic activity may be a result of activation. Considering the accumulation of lymphocytes in the acute phase of AA, our T cell chemotaxis results are consistent with the histopathological findings of $\mathrm{AA}$ in the acute phase [14]. 
2.5. NKG2D in Alopecia Areata. NKG2D is expressed not only in NK cells but also in CD8+ and gamma delta $\mathrm{T}$ cells $[43,44]$. NKG2D recognizes MHC class I chain-related proteins MICA and MICB on target cells. NKG2D also recognizes surface glycoproteins that bind human cytomegalovirus UL16 proteins (ULBPs), from ULBP1 to ULBP6 (total of eight human ligands), which stimulates immune cells to attack target cells [45].

NK cells have become a recent focus of AA research. The absent or low expression of MHC class I in HFs raises the question of how self/nonself-discrimination and selftolerance are maintained [46]. As NK cells recognize and eliminate cells with an absent or low expression of MHC class I [27, 47-49], it is remarkable that very few NK cells gather around the $\mathrm{MHC}$ class I negative human anagen HFs [50]. Just like other healthy tissues, human anagen HFs in situ lack MICA expression [31, 51-53], which may explain why normal HFs are not subject to NK cell attack. However, in AA lesions, infiltrating CD56+ NK cells and CD8+ T cells prominently express NKG2D, and proximal ORS strongly expresses MICA, thus infiltrating NKG2D+ cells attack MICA-positive HFs [31]. Compared with normal controls and patients with other chronic inflammatory skin diseases such as atopic dermatitis, CD56+ NK cells and CD8+ $\mathrm{T}$ cells of AA patients have increased NKG2D expression. In addition, the percentage of NK cells that do not express NK cell-inhibitory KIR2DL2 and KIR2DL3 is significantly increased in AA patients compared with healthy controls [31]. A recent study of 20 families with AA from the United States and Israel has identified genes that may be associated with AA and other autoimmune diseases, such as ULBP genes that encode ligands for activating NKG2D [43].

In summary, immune-privileged, MHC class I-negative HFs are protected from NK cell attack by MICA-negative ORS, low expression of NKG2D on NK cells, and inhibitory KIRs. However, collapse of HFIP leads to NKG2D+ NK cell attack, and autoreactive NKG2D+CD8+ T cells recognize autoantigens that result in apoptotic responses and hair loss in AA.

2.6. Stress Hormone and Alopecia Areata. To maintain homeostasis, the skin must respond to stressors such as ultraviolet light, mechanical injury, and chemical and biological insults. Emotional stress may also perturb skin homeostasis. Components of the hypothalamic-pituitary-adrenal (HPA) axis are present in the skin and are involved in the local response to stress [54]. As part of the HPA axis, corticotropinreleasing hormone $(\mathrm{CRH})$ is a key stress-induced hormone that is present in the human HF. Specifically, $\mathrm{CRH}$ and $\mathrm{CRH}-$ 1 receptors (CRH-R1) are expressed throughout the ORS [55], and CRH and CRH-R1 gene transcriptions occur in the human hair bulb. The human HF has a fully functional peripheral equivalent of the HPA axis, which may be involved in the stress response of the skin [56]. Organ culture human HFs secrete cortisol in response to $\mathrm{CRH}$ and possess feedback systems [56].

Using in situ hybridization, a clinical report examined $\mathrm{CRH}$ receptor expression in three AA patients who had experienced a significant emotional stress prior to hair loss. Skin from the affected scalp areas of all three patients showed an intense signal for CRH-2 $\beta$. Samples from unaffected scalp areas of the same patients or from healthy controls showed only a weak background signal for the receptor [57]. The expression of $\mathrm{CRH}, \mathrm{ACTH}$, and $\alpha$-MSH was significantly increased in the epidermis, HFs, and sebaceous glands of samples from AA patients compared with those from healthy controls. These results suggest the presence of an active neurogenic system and local HPA activity in AA lesions [57]. Immune activation not only affects $\mathrm{CRH}$ expression but also HPA activity [58-64].

Central and peripheral HPA activity under basal and stressful conditions were investigated in normal and AAaffected $\mathrm{C} 3 \mathrm{H} / \mathrm{HeJ}$ mice [65]. In response to psychological stress, normal mice showed marked plasma corticosterone elevation, whereas AA-affected mice showed no significant changes in corticosterone levels, suggesting that AA-affected mice have a blunted response to acute physiological stress.

In conclusion, emotional stress may affect AA patients because of a blunted corticosterone response to hormones and immunological damage, and altered HPA activity may occur as a consequence of the immune response associated with AA.

\section{Conclusion}

The HF is a dynamic "miniorgan" with unique immune and hormone microenvironments. Immune privilege is the most intriguing feature of HF immunology that is characterized by the downregulation of MHC class I. The collapse of HFIP is induced by certain environmental factors such as virus infections, and it involves the production of the Th1/Tc1 chemokine, CXCL10, in HFs that attracts Th1 and Tcl cells towards the hair bulbs. Consequently, HF autoantigens are recognized by autoreactive cytotoxic T cells. HPA activity in the HF links the immune and hormonal aspects of the HF. Environmental stress may influence both the immune and hormonal microenvironments of the $\mathrm{HF}$ and result in the development of AA. Patients with AA may have a blunted response to an acute physiological stressor, resulting in a reduced expression of glucocorticoids.

Recent advances in the understanding of the pathomechanism of AA may lead to the development of novel treatments for $\mathrm{AA}$ in the future.

\section{Acknowledgments}

The author thanks Dr. N. Ito, Dr. M. Bertolini, Dr. E. Bodò, Professor R. Paus, and Professor Y. Tokura for their contributions to the research of alopecia areata.

\section{References}

[1] V. K. Sharma, G. Dawn, and B. Kumar, "Profile of alopecia areata in Northern India," International Journal of Dermatology, vol. 35, no. 1, pp. 22-27, 1996.

[2] E. Tan, Y.-K. Tay, C.-L. Goh, and Y. C. Giam, "The pattern and profile of alopecia areata in Singapore-a study of 219 Asians," 
International Journal of Dermatology, vol. 41, no. 11, pp. 748-753, 2002.

[3] D. Wasserman, D. A. Guzman-Sanchez, K. Scott, and A. Mcmichael, "Alopecia areata," International Journal of Dermatology, vol. 46, no. 2, pp. 121-131, 2007.

[4] A. Alkhalifah, A. Alsantali, E. Wang, K. J. McElwee, and J. Shapiro, "Alopecia areata update. Part I. Clinical picture, histopathology, and pathogenesis," Journal of the American Academy of Dermatology, vol. 62, no. 2, pp. 177-188, 2010.

[5] M. Sato-Kawamura, S. Aiba, and H. Tagami, "Acute diffuse and total alopecia of the female scalp. A new subtype of diffuse alopecia areata that has a favorable prognosis," Dermatology, vol. 205, no. 4, pp. 367-373, 2002.

[6] B.-L. Lew, M.-K. Shin, and W.-Y. Sim, "Acute diffuse and total alopecia: a new subtype of alopecia areata with a favorable prognosis," Journal of the American Academy of Dermatology, vol. 60, no. 1, pp. 85-93, 2009.

[7] H. Seyrafi, M. Akhiani, H. Abbasi, S. Mirpour, and A. Gholamrezanezhad, "Evaluation of the profile of alopecia areata and the prevalence of thyroid function test abnormalities and serum autoantibodies in Iranian patients," BMC Dermatology, vol. 5, article 11, 2005.

[8] E. Kasumagić-Halilović, "Thyroid autoimmunity in patients with alopecia areata," Acta Dermatovenerologica Croatica, vol. 16, pp. 123-125, 2008.

[9] M. Hordinsky and M. Ericson, "Autoimmunity: alopecia areata," Journal of Investigative Dermatology Symposium Proceedings, vol. 9, no. 1, pp. 73-78, 2004.

[10] S. Ruiz-Doblado, A. Carrizosa, and M. J. García-Hernández, "Alopecia areata: psychiatric comorbidity and adjustment to illness," International Journal of Dermatology, vol. 42, no. 6, pp. 434-437, 2003.

[11] E. A. Colón, M. K. Popkin, A. L. Callies, N. J. Dessert, and M. K. Hordinsky, "Lifetime prevalence of psychiatric disorders in patients with alopecia areata," Comprehensive Psychiatry, vol. 32, no. 3, pp. 245-251, 1991.

[12] D. Weedon, "Diseases of cutaneous appendages," in Weedon's Skin Pathology, D. Weedon, Ed., Churchill Livingstone, London, UK, 2002.

[13] N. Todes-Taylor, R. Turner, and G. S. Wood, “T cell subpopulations in alopecia areata," Journal of the American Academy of Dermatology, vol. 11, no. 2, pp. 216-223, 1984.

[14] T. Ito, H. Hashizume, T. Shimauchi et al., "CXCL10 produced from hair follicles induces Thl and Tcl cell infiltration in the acute phase of alopecia areata followed by sustained Tc1 accumulation in the chronic phase," Journal of Dermatological Science, vol. 69, pp. 140-147, 2013.

[15] L. Stankler, "Synchronous alopecia areata in two siblings: a possible viral aetiology," Lancet, vol. 1, no. 8129, pp. 1303-1304, 1979.

[16] A. Akar, E. Arca, H. Erbil, C. Akay, A. Sayal, and A. R. Gür, "Antioxidant enzymes and lipid peroxidation in the scalp of patients with alopecia areata," Journal of Dermatological Science, vol. 29, no. 2, pp. 85-90, 2002.

[17] B. W. Colombe, V. H. Price, E. L. Khoury, M. R. Garovoy, and C. D. Lou, "HLA class II antigen associations help to define two types of alopecia areata," Journal of the American Academy of Dermatology, vol. 33, no. 5 I, pp. 757-764, 1995.

[18] M. De Andrade, C. M. Jackow, N. Dahm, M. Hordinsky, J. D. Reveille, and M. Duvic, "Alopecia areata in families: association with the HLA locus," Journal of Investigative Dermatology Symposium Proceedings, vol. 4, no. 3, pp. 220-223, 1999.
[19] E. A. Welsh, H. H. Clark, S. Z. Epstein, J. D. Reveille, and M. Duvic, "Human leukocyte Antigen-DQB1" 03 alleles are associated with alopecia areata," Journal of Investigative Dermatology, vol. 103, no. 6, pp. 758-763, 1994.

[20] N. Morling, G. Frentz, L. Fugger et al., "DNA polymorphism of HLA class II genes in alopecia areata," Disease Markers, vol. 9, no. 1, pp. 35-42, 1991.

[21] L. E. King Jr., K. J. McElwee, and J. P. Sundberg, "Alopecia areata," Current Directions in Autoimmunity, vol. 10, pp. 280312, 2008.

[22] L. Petukhova, M. Duvic, M. Hordinsky et al., "Genome-wide association study in alopecia areata implicates both innate and adaptive immunity," Nature, vol. 466, no. 7302, pp. 113-117, 2010.

[23] R. Paus, B. J. Nickoloff, and T. Ito, "A 'hairy' privilege," Trends in Immunology, vol. 26, no. 1, pp. 32-40, 2005.

[24] T. Ito, K. Meyer, N. Ito, and R. Paus, "Immune privilege and the skin," Current Directions in Autoimmunity, vol. 10, pp. 27-52, 2008.

[25] T. Ito, "Hair follicle is a target of stress hormone and autoimmune reactions," Journal of Dermatological Science, vol. 60, no. 2, pp. 67-73, 2010.

[26] T. Ito, N. Ito, M. Saathoff, A. Bettermann, M. Takigawa, and R. Paus, "Interferon- $\gamma$ is a potent inducer of catagen-like changes in cultured human anagen hair follicles," British Journal of Dermatology, vol. 152, no. 4, pp. 623-631, 2005.

[27] T. Ito and Y. Tokura, "Alopecia areata triggered or exacerbated by swine flu virus infection," Journal of Dermatology, vol. 39, pp. 863-864, 2012.

[28] R. Paus, A. Slominski, and B. M. Czarnetzki, "Is alopecia areata an autoimmune-response against melanogenesis-related proteins, exposed by abnormal MHC class I expression in the anagen hair bulb?" Yale Journal of Biology and Medicine, vol. 66, no. 6, pp. 541-554, 1993.

[29] A. Gilhar, M. Landau, B. Assy, R. Shalaginov, S. Serafimovich, and R. S. Kalish, "Melanocyte-associated T cell epitopes can function as autoantigens for transfer of alopecia areata to human scalp explants on Prkdcacid mice," Journal of Investigative Dermatology, vol. 117, no. 6, pp. 1357-1362, 2001.

[30] D. J. Tobin, D. A. Fenton, and M. D. Kendall, "Ultrastructural observations on the hair bulb melanocytes and melanosomes in acute alopecia areata," Journal of Investigative Dermatology, vol. 94, no. 6, pp. 803-807, 1990.

[31] T. Ito, N. Ito, M. Saatoff et al., "Maintenance of hair follicle immune privilege is linked to prevention of NK cell attack," Journal of Investigative Dermatology, vol. 128, no. 5, pp. 11961206, 2008.

[32] E. Arca, U. Muşabak, A. Akar, A. H. Erbil, and H. B. Taştan, "Interferon-gamma in alopecia areata," European Journal of Dermatology, vol. 14, no. 1, pp. 33-36, 2004.

[33] A. Gilhar, Y. Kam, B. Assy, and R. S. Kalish, "Alopecia areata induced in $\mathrm{C} 3 \mathrm{H} / \mathrm{HeJ}$ mice by interferon-gamma: evidence for loss of immune privilege," Journal of Investigative Dermatology, vol. 124, no. 1, pp. 288-289, 2005.

[34] R. Gillitzer, S. Benoit, A. Toksoy, and M. Goebeler, "Selective expression of chemokine monokine induced by interferon $-\gamma$ in alopecia areata," Journal of Investigative Dermatology, vol. 121, no. 4, pp. 933-935, 2003.

[35] A. Gilhar, M. Landau, B. Assy et al., “Transfer of alopecia areata in the human scalp graft/Prkdcscid (SCID) mouse system is characterized by a TH1 response," Clinical Immunology, vol. 106, no. 3, pp. 181-187, 2003. 
[36] Y. Kuwano, M. Fujimoto, R. Watanabe et al., "Serum chemokine profiles in patients with alopecia areata," British Journal of Dermatology, vol. 157, no. 3, pp. 466-473, 2007.

[37] R. D. Subramanya, A. B. Coda, and A. A. Sinha, "Transcriptional profiling in alopecia areata defines immune and cell cycle control related genes within disease-specific signatures," Genomics, vol. 96, no. 3, pp. 146-153, 2010.

[38] T. R. Mosmann, L. Li, and S. Sad, "Functions of CD8 Tcell subsets secreting different cytokine patterns," Seminars in Immunology, vol. 9, no. 2, pp. 87-92, 1997.

[39] S. Sad, R. Marcotte, and T. R. Mosmann, "Cytokine-induced differentiation of precursor mouse $\mathrm{CD}^{+} \mathrm{T}$ cells into cytotoxic $\mathrm{CD}^{+} \mathrm{T}$ cells secreting Th1 or Th2 cytokines," Immunity, vol. 2 , no. 3, pp. 271-279, 1995.

[40] T. R. Mosmann and S. Sad, "The expanding universe of T-cell subsets: Th1, Th2 and more," Immunology Today, vol. 17, no. 3, pp. 138-146, 1996.

[41] Y. Yu, H. I. Cho, D. Wang et al., "Adoptive transfer of Tc1 or Tc17 cells elicits antitumor immunity against established melanoma through distinct mechanisms," The Journal of Immunology, vol. 190, pp. 1873-1881, 2013.

[42] M.-P. Brazillet, F. Batteux, O. Abehsira-Amar, F. Nicoletti, and J. Charreire, "Induction of experimental autoimmune thyroiditis by heat-denatured porcine thyroglobulin: a Tc1-mediated disease," European Journal of Immunology, vol. 29, no. 4, pp. 13421352, 1999.

[43] B. Zafirova, F. M. Wensveen, M. Gulin, and B. Polić, "Regulation of immune cell function and differentiation by the NKG2D receptor," Cellular and Molecular Life Sciences, vol. 68, no. 21, pp. 3519-3529, 2011.

[44] D. H. Raulet, S. Gasser, B. G. Gowen, W. Deng, and H. Jung, "Regulation of ligands for the NKG2D activating receptor," Annual Review of Immunology, vol. 31, pp. 413-441, 2013.

[45] S. Bauer, V. Groh, J. Wu et al., "Activation of NK cells and T cells by NKG2D, a receptor for stress- inducible MICA," Science, vol. 285, no. 5428, pp. 727-729, 1999.

[46] T. Boehm, "Quality control in self/nonself discrimination," Cell, vol. 125, no. 5, pp. 845-858, 2006.

[47] W. M. Yokoyama and S. Kim, "Licensing of natural killer cells by self-major histocompatibility complex class I," Immunological Reviews, vol. 214, no. 1, pp. 143-154, 2006.

[48] S. Gasser and D. H. Raulet, "Activation and self-tolerance of natural killer cells," Immunological Reviews, vol. 214, no. 1, pp. 130-142, 2006.

[49] Y. T. Bryceson, M. E. March, H.-G. Ljunggren, and E. O. Long, "Activation, coactivation, and costimulation of resting human natural killer cells," Immunological Reviews, vol. 214, no. 1, pp. 73-91, 2006.

[50] T. Christoph, S. Müller-Röver, H. Audring et al., "The human hair follicle immune system: cellular composition and immune privilege," British Journal of Dermatology, vol. 142, no. 5, pp. 862-873, 2000.

[51] L. Deng and R. A. Mariuzza, "Structural basis for recognition of MHC and MHC-like ligands by natural killer cell receptors," Seminars in Immunology, vol. 18, no. 3, pp. 159-166, 2006.

[52] V. Groh, A. Steinle, S. Bauer, and T. Spies, "Recognition of stressinduced MHC molecules by intestinal epithelial $\gamma \delta$ T cells," Science, vol. 279, no. 5357, pp. 1737-1740, 1998.

[53] V. Groh, R. Rhinehart, H. Secrist, S. Bauer, K. H. Grabstein, and T. Spies, "Broad tumor-associated expression and recognition by tumor-derived $\gamma \delta$ T cells of MICA and MICB," Proceedings of the National Academy of Sciences of the United States of America, vol. 96, no. 12, pp. 6879-6884, 1999.

[54] A. Slominski, J. Wortsman, T. Luger, R. Paus, and S. Solomon, "Corticotropin releasing hormone and proopiomelanocortin involvement in the cutaneous response to stress," Physiological Reviews, vol. 80, no. 3, pp. 979-1020, 2000.

[55] P. C. Arck, B. Handjiski, E. M. J. Peters et al., "Stress inhibits hair growth in mice by induction of premature catagen development and deleterious perifollicular inflammatory events via neuropeptide substance P-dependent pathways," American Journal of Pathology, vol. 162, no. 3, pp. 803-814, 2003.

[56] N. Ito, T. Ito, A. Kromminga et al., "Human hair follicles display a functional equivalent of the hypothalamic-pituitary-adrenal axis and synthesize cortisol," FASEB Journal, vol. 19, no. 10, pp. 1332-1334, 2005.

[57] A. Katsarou-Katsari, L. K. Singh, and T. C. Theoharides, "Alopecia areata and affected skin CRH receptor upregulation induced by acute emotional stress," Dermatology, vol. 203, no. 2, pp. 157-161, 2001.

[58] H. S. Kim, D. H. Cho, H. J. Kim, J. Y. Lee, B. K. Cho, and H. J. Park, "Immunoreactivity of corticotropin-releasing hormone, adrenocorticotropic hormone and $\alpha$-melanocyte-stimulating hormone in alopecia areata," Experimental Dermatology, vol. 15, no. 7, pp. 515-522, 2006.

[59] S. A. Muller and R. K. Winkelmann, "Alopecia areata. An evaluation of 736 patients," Archives of Dermatology, vol. 88, pp. 290-297, 1963.

[60] J. J. Jessop, K. Gale, and B. M. Bayer, "Enhancement of rat lymphocyte proliferation after prolonged exposure to stress," Journal of Neuroimmunology, vol. 16, no. 2, pp. 261-271, 1987.

[61] N. Shanks, M. S. Harbuz, D. S. Jessop, P. Perks, P. M. Moore, and S. L. Lightman, "Inflammatory disease as chronic stress," Annals of the New York Academy of Sciences, vol. 840, pp. 599-607, 1998.

[62] I. J. Elenkov and G. P. Chrousos, "Stress hormones, Th1/Th2 patterns, pro/anti-inflammatory cytokines and susceptibility to disease," Trends in Endocrinology and Metabolism, vol. 10, no. 9, pp. 359-368, 1999.

[63] I. J. Elenkov and G. P. Chrousos, "Stress hormones, proinflammatory and antiinflammatory cytokines, and autoimmunity," Annals of the New York Academy of Sciences, vol. 966, pp. 290303, 2002.

[64] M. S. Harbuz, L. J. Richards, A. J. Chover-Gonzalez, O. MartiSistac, and D. S. Jessop, "Stress in autoimmune disease models," Annals of the New York Academy of Sciences, vol. 1069, pp. 51-61, 2006.

[65] X. Zhang, M. Yu, W. Yu, J. Weinberg, J. Shapiro, and K. J. McElwee, "Development of alopecia areata is associated with higher central and peripheral hypothalamic-pituitary-adrenal tone in the skin graft induced C3HHeJ mouse model," Journal of Investigative Dermatology, vol. 129, no. 6, pp. 1527-1538, 2009. 


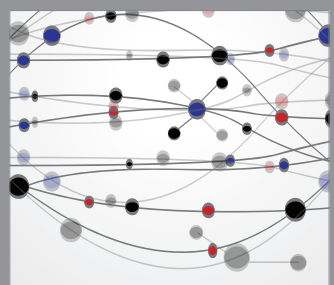

The Scientific World Journal
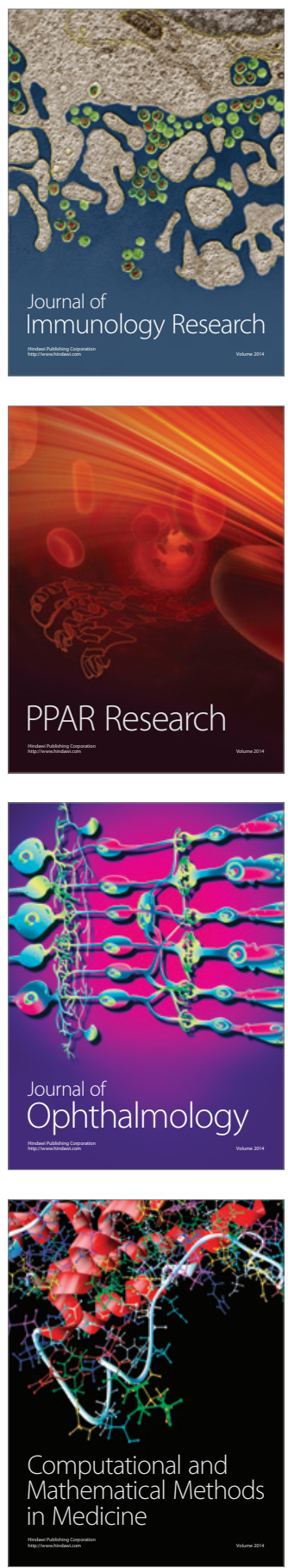

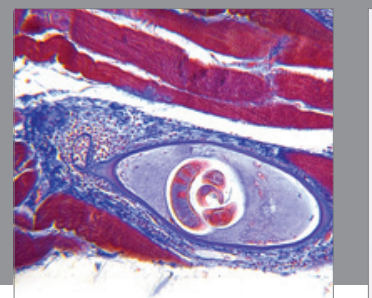

Gastroenterology

Research and Practice
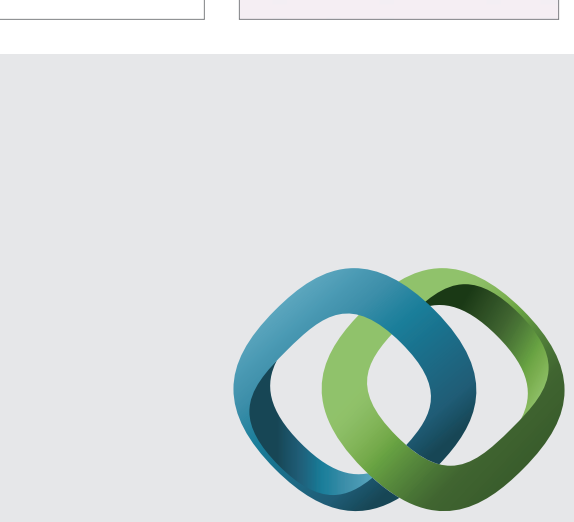

\section{Hindawi}

Submit your manuscripts at

http://www.hindawi.com
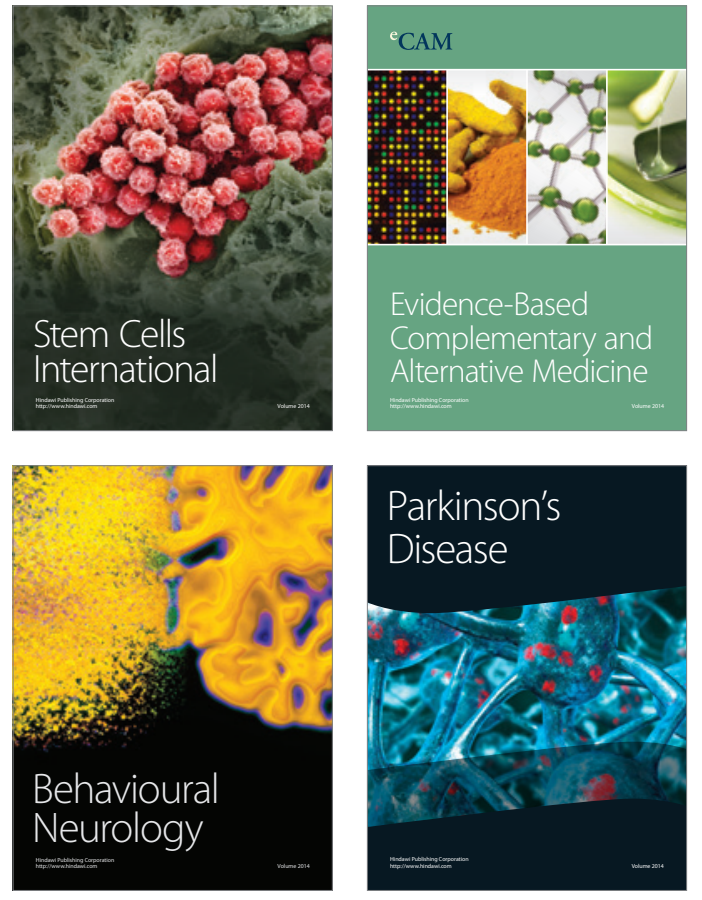
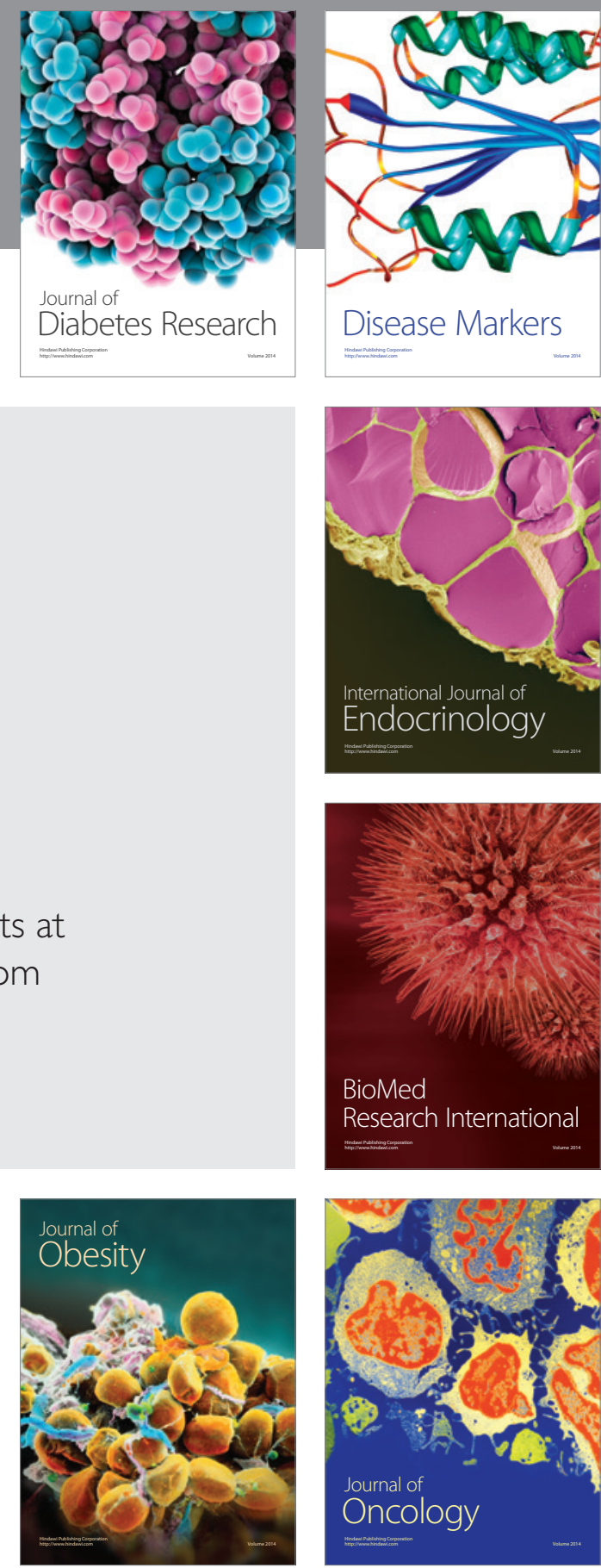

Disease Markers
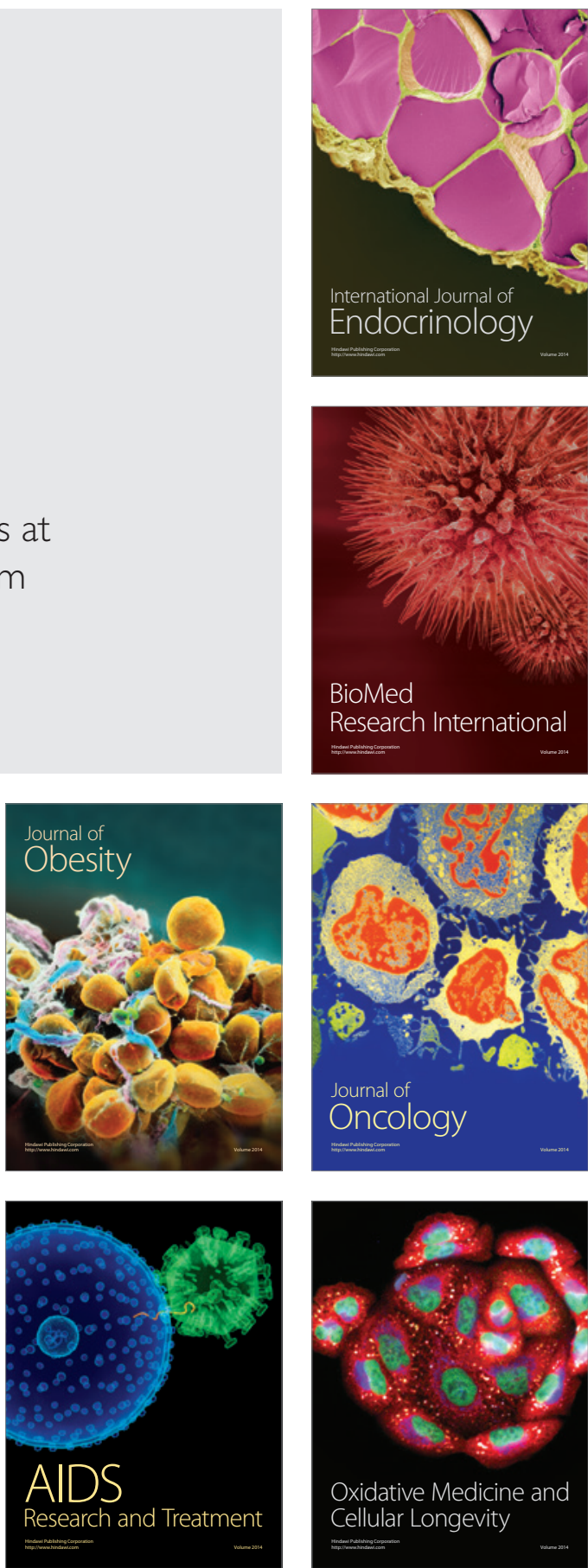